Using short pulses $(0.5 \mathrm{msec})$, enough echo strength was available from the planetary surface several pulse widths behind the nearest point to measure the Doppler frequency spectrum at known relative delays.

The procedure is illustrated in figure 1, showing the two humps in the frequency spectrum which arise because of the larger surface area lying at these values of range and frequency. The limb-to-limb Doppler difference and the instantaneous apparent rotation can be computed from the separation in frequency at given time or distance after the maximum echo. An estimate of error in the reading of the data can be obtained from the consistency of several limb-to-limb determinations on a given date.

It is clearly indicated that the accepted synchronous rotation does not fit the radar observations (see review paper in this same issue). The best estimate appears to be a sidereal rotation period of $59 \pm 5$ days in a direct sense. Fortunately, the measurements can be repeated in August 1965 at a different place along the Mercury orbit.

(Paper 69D12-612)

\title{
Recent Arecibo Observations of Mars and Jupiter
}

\author{
R. B. Dyce \\ Arecibo Ionospheric Observatory, ${ }^{1}$ Arecibo, Puerto Rico
}

Mars has been under observation at Arecibo since 19 November 1964 but the strongest returns were obtained during the month of March 1965. The relatively wide frequency spectrum (1300 c/s limb-to-limb) does not permit simultaneous determination of Doppler spectrum and range.

During the most favorable observing period of 1965 the subradar point on Mars traversed a line of Martian latitude of $+21^{\circ}$. The radar reflectivity from the surface varies widely $(0.03$ to $0.13 \pi r^{2}$ ) as a function of longitude (see fig. 1). There is a tendency for the strongest echo strength to be associated with dark regions passing near the subradar point, with the remarkable exception of a notch exactly where the tip of Syrtius Major passes the subradar point $\left(277^{\circ}\right)$. The JPL reflectivity variation of 1963 appropriate to a subradar latitude of $+13^{\circ}$ shows some similarity. Results from JPL 1964 confirm the AIO curve, especially the reflectivity peak near Trivium Charontis.

${ }^{1}$ Operated by Cornell University with the support of the Advanced Research Projects Agency under a research contract with the Air Force Office of Scientific Research, OAR.

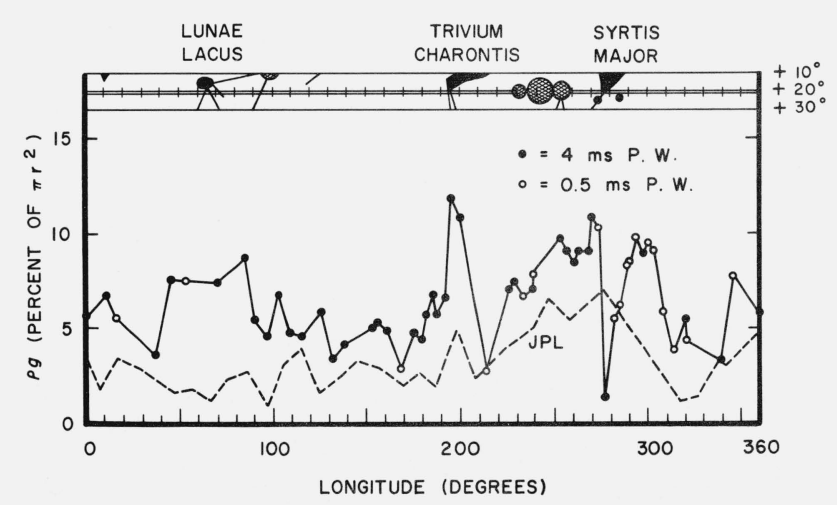

FigurE 1. The 430-Mc/s radar reflectivity of Mars as a function of the longitude of the subradar point, showing the large variability. There is a tendency for high values of radar reflectivity to be associated with the dark regions on Mars (shown in the strip map at the top of the figure). 
Particularly interesting is the presence of both a stable, broadband frequency component and a variable narrowband component that is associated with longitudes of high reflectivity (see review paper for implications on surface characteristics).

In the fall of 1964 radar observations of Jupiter were attempted at a wavelength of $70 \mathrm{~cm}$. No significant echoes were observed.

The assistance of A. D. Sanchez and D. P. Campbell is specifically acknowledged.

Discussion following R. B. Dyce's paper

$J$. Warwick: Can these negative observations be expressed, in the absence of absorption, as an upper limit to the Jovian ionospheric electron density?

R. B. Dyce: Yes. A critical reflection model would set an upper limit of $2 \times 10^{9}$ electrons $-\mathrm{cm}^{-3}$.

J. Warwick: Are there plans to employ the future 40-Mc/s Arecibo radar system to such a search for a Jovian ionosphere?

G. H. Pettengill: The lower antenna gain and higher sky noise will cause the $40-\mathrm{Mc} / \mathrm{s}$ system to be about $20 \mathrm{~dB}$ less sensitive than the $430-\mathrm{Mc} / \mathrm{s}$ system.

R. Goldstein: No Jupiter echoes were detected by the JPL equipment in 1964 using an identical experimental configuration as in 1963.

(Paper 69D12-613)

\title{
Radio Evidence on the Structure and Composition of the Martian Surface
}

\author{
Carl Sagan \\ Harvard University \\ and \\ Smithsonian Astrophysical Observatory, Cambridge, Mass. \\ and \\ James B. Pollack \\ Smithsonian Astrophysical Observatory, Cambridge, Mass.
}

The radar observations of Mars near the 1963 and 1965 oppositions are discussed. In the 1963 observations of Goldstein and Gillmore, near planetocentric latitude $13^{\circ}$ north, a significant correlation is noted of radar reflectivity enhancements with the Martian dark areas, Cerberus Trivium Charontis, Nepenthes, and Syrtis Major. Limonite, $\mathrm{Fe}_{2} \mathrm{O}_{3} \cdot n \mathrm{H}_{2} \mathrm{O}$, is suspected to be a predominant constituent of the Martian bright areas on the basis of a variety of colorimetric, polarimetric, and infrared spectrometric observations. The values of the general radar reflectivity of the Martian bright areas are consistent with the identification of limonite on Mars only if the limonite is pulverized down to a depth of some meters. The deduced degree of pulverization of Martian limonite has been found to be achieved easily by pulverized limonite in the laboratory. The Martian dark areas are probably porous to a depth of meters, but their porosity is less than that of the bright areas. If limonite is a major constituent of the Martian bright areas, then passive microwave observations at wavelengths longward of $1 \mathrm{~cm}$ refer to a depth at which the diurnal thermal wave is damped to negligibly small amplitude. The mutual consistency of the infrared and microwave brightness temperatures of Mars is discussed.

(Paper 69D12-614) 